DOI: $10.5902 / 198346598475$

\title{
PREÇO E RENDA COMO DETERMINANTES DA DEMANDA POR BENS DE LUXO NO BRASIL: UM ESTUDO ECONOMÉTRICO COM PRODUTOS IMPORTADOS DA NOMENCLATURA COMUM DO MERCOSUL
}

\author{
PRICE AND INCOME AS DETERMINANTS OF THE DEMAND FOR \\ LUXURY GOODS IN BRAZIL: AN ECONOMETRIC STUDY WITH \\ IMPORTS FROM THE MERCOSUR COMMON NOMENCLATURE
}

\author{
Data de submissão: 29/03/2013 \\ Aceite: 05/04/2014 \\ Paulo Roberto Scalco ${ }^{1}$ \\ Mariana Klaold Lippi ${ }^{2}$ \\ Marcos Inácio Severo de Almeida ${ }^{3}$
}

\section{RESUMO}

Este artigo oferece uma abordagem alternativa ao promover a análise dos determinantes da demanda por produtos de luxo a partir de dados de importação de mais de mil bens da tabela de produtos da Nomenclatura Comum do Mercosul (NCM). Com base no volume de importações entre janeiro de 2000 e março de 2011, foram testadas duas hipóteses sobre os determinantes da demanda: preço e renda. Os resultados revelam que apenas a renda é estatisticamente significante na determinação da demanda. Esses resultados estão de acordo com o que se discute na teoria econômica, que define como bens de luxo aqueles cuja elasticidade-renda da demanda é superior a um. A não significância estatística do fator preço pode ser consequência de uma limitação metodológica, em função da proxy utilizada, ou pode ser resultado das características da demanda não funcional: consumidores estariam dispostos a adquirir esses bens mesmo diante de preços mais elevados.

Palavras-chave: Bens de luxo; Consumo; Demanda; Renda; Taxa de câmbio real

\footnotetext{
${ }^{1}$ Possui graduação em Ciências Econômicas pela Universidade de Passo Fundo, UPF, mestrado em Economia pela Universidade Federal de Viçosa, UFV e doutorado em Economia Aplicada pela Universidade Federal de Viçosa, UFV. Goiânia. Goiás. Brasil. E-mail: pauloscalco@ yahoo.com.br

${ }^{2}$ Possui graduação em Ciências Econômicas pela Universidade Federal de Goiás, UFG. Goiânia. Goiás. Brasil. E-mail: marianaklahold@ gmail.com

${ }^{3}$ Possui graduação em Comunicação pela Pontifícia Universidade Católica do Rio de Janeiro, PUC-Rio, mestrado em Administração pela Universidade Federal de Viçosa, UFV e doutorado em andamento em Administração pela Universidade de Brasília, UnB. Goiânia. Goiás. Brasil. E-mail: misevero@yahoo.com.br
} 


\begin{abstract}
This article offers an alternative approach as promote an analysis on the determinants of demand for luxury products with data of more than a thousand imported products of the table of Mercosur Common Nomenclature (NCM). Through the volume of imports between January 2000 and March 2011 two hypotheses were tested about the determinants of demand: price and income. The results show that only income is statistically significant in determining the demand. These results are in agreement with what is discussed in economic theory, which defines luxury goods as those whose income elasticity of demand is greater than one. The non-statistical significance of the price factor may be result of a methodological limitation due to the use of the proxy for price or may be the result of non-functional characteristics of demand: consumers of luxury purchase them even in the face of higher prices.
\end{abstract}

Keywords: Luxury goods; Consumption; Demand; Income; Exchange rate

\title{
1 INTRODUÇÃO
}

Desde o lançamento do Plano Real em 1994, o cenário econômico brasileiro passou por diversas transformações. A proposta do novo sistema monetário foi acompanhada de um processo generalizado de desregulação, abertura econômica e estabilização do nível de preços. Essas transformações colocaram o país diante de uma estabilidade que há décadas não se observava. Como efeitos, houve queda e controle da inflação, redução da desigualdade e dos níveis de pobreza e aumento da renda e do bem-estar social (FERREIRA et al., 2006; FIGUEIREDO; SILVA, 2012; NERI, 2006). Essa nova realidade proporcionou aumento no consumo da população brasileira, principalmente no que se refere à procura por uma categoria específica de produto, classificada como bem de luxo, e colocou o Brasil entre os seis países com gastos mais representativos nesse mercado (BELLACHE; MEI-POCHTLER; HANISCH, 2010).

Segundo um estudo conduzido pelo The Boston Consulting Group, o luxo possui diversos significados para diferentes indivíduos. A terminologia é utilizada de maneira genérica para definir produtos, serviços ou estilos de vida, e sua conceituação também depende de questões relacionadas ao humor e à experiência do consumidor (BELLAICHE; MEI-POCHTLER; HANISCH, 2010; WIEDMANN; HENNIGS; SIEBELS, 2007). De acordo com Pereira, Bido e Kimura (2009), trata-se de um segmento de mercado no qual estilo e dimensões simbólicas são reconhecidamente importantes e em que o preço não representa uma condição necessária, apenas suficiente, para definir um produto como um bem de luxo. Um pressuposto aceito, no entanto, é o de que o termo está relacionado a características como raridade, qualidade e refinamento, benefícios normalmente oferecidos por produtos e serviços como automóveis, bebidas alcoólicas, joias, relógios, vestuários, turismo e hotelaria (BELLAICHE; MEI-POCHTLER; HANISCH, 2010).

Para Allérès (2000), um objeto de luxo deve reunir certos atributos, como qualidade, marca universal, distribuição seletiva, comunicação adequada e preços mais elevados. Esse autor comenta que criações inusitadas e que são sinônimos de beleza, estética e refinamento se caracterizam como objetos de luxo. Na tentativa de esclarecer o significado da terminologia, Kapferer (2012) destaca a existência de três dimensões: 1) faz referência a um produto raro, de preço alto, hedônico e de qualidade percebida; 2 ) trata-se de um setor econômico, que reúne organizações específicas que movimentam bilhões de euros por meio da comercialização de produtos ou serviços; e 3) refere-se também a um modelo de negócio, que prevê o uso de estratégias para se conquistar determinado mercado-alvo.

É importante acrescentar uma questão comportamental ao modelo de Kapferer (2012): o consumo de bens de luxo acontece porque os indivíduos se preocupam em atender necessida- 
des psicológicas com algo exclusivo e em comunicar significados para seus grupos de referência (GROTH, 1994; HUSIC; CICIC, 2009). No consumo desse gênero de bens, parte da demanda é estimulada por fatores exógenos aos atributos do produto. Normalmente, são duas as alternativas possíveis manifestadas pelo adquirente: apresenta o que se define como efeito ou fator esnobe, uma ação de compra cujo objetivo é distingui-lo dos demais por meio da atribuição de respeito e prestígio, em função do caráter exclusivo do produto; ou exibe o que se nomeia de efeito Veblen, quando sinaliza riqueza por meio da aquisição de um produto cujo preço é alto. O que diferencia os dois fenômenos é que o primeiro se relaciona com o consumo de terceiros, enquanto que o último tem ligação direta com o preço (GUPTA, 2009; LEIBENSTEIN, 1950).

Giacalone (2006) comenta que economistas consideram as características particulares do consumo de bens de luxo desde o trabalho de Veblen (1899) e sua caracterização do consumo conspícuo como modo de aquisição de bens e serviços para ostentação de status e riqueza. Esforços conduzidos posteriormente na literatura específica da Administração Mercadológica orientaram o desenvolvimento de estudos que evidenciaram outros fatores capazes de influenciar o consumo de bens de luxo, além da renda proveniente do trabalho - o income - e da riqueza (a soma de todos os ganhos de um agente), duas variáveis normalmente definidas em modelos microeconômicos (GIACALONE, 2006). Observam-se, nesse campo de pesquisa, investigações conduzidas a partir de diferentes abordagens, como aquelas que procuram relacionar renda e fatores culturais ao consumo (DUBOIS; DUQUESNE, 1993; SHUKLA; PURANI, 2012), identificar dimensões da percepção individual do luxo (SHUKLA, 2012; WIEDMANN; HENNIGS; SIEBELS, 2007), identificar fatores determinantes para o consumo de bens de luxo (HUDDERS, 2012; HUSIC; CICIC, 2009), compreender os motivos subjacentes ao uso de produtos de luxo e falsificados (WIEDMANN; HENNIGS; KLARMANN, 2012) e, inclusive, apresentar quadro conceitual para o consumo desse gênero de produtos em países sob ameaça terrorista (SULEHRI et al., 2011).

Em termos acadêmico-gerenciais, Wiedmann e Hennigs (2013) destacam a existência de quatro abordagens que pavimentam o caminho para futuras pesquisas em marketing sobre o luxo. A primeira delas é a abordagem classificada como commodity, que questiona quais são os tipos de produtos que podem ser definidos como luxuosos. Em seguida, está a percepção do consumidor, que enfatiza o luxo como conceito subjetivo, procurando identificar as dimensões do luxo percebidas por consumidores em nível individual. A terceira abordagem consiste na visão gerencial do luxo, que se volta para os objetivos e as diretrizes mercadológicas que diferenciam marcas exclusivas de outras marcas, baseando-se em critérios como exclusividade e preço premium. Por fim, há a abordagem funcional ou instrumental, que se dedica a identificar os direcionadores responsáveis por criar uma experiência luxuosa.

No Brasil, o luxo como temática recebe atenção de pesquisadores, embora a quantidade de estudos ainda seja escassa, principalmente quando comparada a outras áreas do campo do conhecimento em marketing. Uma característica marcante do delineamento das produções brasileiras é a concentração no processo de decisão de compra, na percepção ou em características experienciais do consumo do luxo. Outra consideração importante é uma predominância de investigações exploratórias e qualitativas, publicadas, em grande parte, em anais de encontros científicos, que se valem de abordagens metodológicas como o estudo de caso e técnicas como entrevistas (BRUNELLI et al., 2012; FERREIRA et al., 2013; FINESTRALLI; GARRIDO, 2008; MOUSINHO; PEREIRA; STORNI, 2010; PEREIRA; BIDO; KIMURA, 2010; SÁ; MARCONDES, 2010). As discussões teóricas sobre o valor da marca no Brasil não consideram a importância do tema (REGO; OLIVEIRA; LUCE, 2008), ao mesmo tempo em que poucos são os trabalhos publicados em periódicos que utilizam metodologias sistemáticas, longitudinais, quantitativas e que direcionam 
a investigação para questões como renda e preço como fatores significativos para a aquisição de bens de luxo no país. Pinto e Lara (2008) foram categóricos ao apontar o baixo quantitativo de estudos causais sobre consumo (aproximadamente 15\%, entre 1997 e 2006) e menos ainda de estudos que incorporassem dados secundários para delimitar questões de pesquisa.

Diante do exposto, o presente artigo oferece uma abordagem alternativa para a compreensão do tema ao apresentar um estudo econométrico com dados de importações de mais de mil bens, escolhidos após um levantamento na tabela de produtos da Nomenclatura Comum do Mercado Comum do Sul (Mercosul) (NCM). A justificativa para a escolha dessa cesta de bens como base de dados se deve ao fato de não existirem características definidoras de um produto de luxo nos campos do marketing e da microeconomia. Na microeconomia, especificamente, a definição de um bem de luxo limita-se a fazer referência a sua elasticidade-renda da demanda como sendo superior a um. Ou seja, o orçamento doméstico gasto com esse gênero de bem varia positivamente e mais que proporcionalmente em relação à variação da renda familiar (KEMP, 1998; VARIAN, 2009). Da mesma forma, no âmbito do marketing, pesquisadores que se destinaram a investigar esse fenômeno destacam a dificuldade de definição, identificação ou categorização dos bens de luxo (KAPFERER, 2012; WIEDMANN; HENNIGS; SIEBELS, 2007).

Para atingir o objetivo de analisar preço e renda como fatores determinantes da demanda por produtos de luxo importados pelo Brasil, duas hipóteses foram testadas, relativas às influências dessas duas variáveis independentes. A taxa de câmbio real foi definida como proxy do preço, e o Produto Interno Bruto (PIB) foi estipulado como proxy da renda. Os dados coletados para a operacionalização do estudo econométrico foram os da série temporal das importações compreendidas no período de janeiro de 2000 a março de 2011, acompanhados dos respectivos dados da taxa de câmbio real e do PIB nesse período. O método de Mínimos Quadrados Ordinários (MQO) foi utilizado para estimar parâmetros de modelos econométricos e realizar testes de hipóteses relativos a restrições lineares no vetor de parâmetros. Os resultados indicam que apenas a renda é estatisticamente significativa na determinação da demanda por esses bens.

Este artigo se estrutura em seis seções, além desta introdução. A segunda seção apresenta uma breve contextualização sobre o luxo no cenário econômico atual, acompanhada da construção das hipóteses de pesquisa. Em seguida, apresentam-se o modelo que orientou a investigação da demanda por importações e os procedimentos adotados para a análise dos dados. Já na quarta seção, são descritas as variáveis utilizadas, enquanto que, na seção cinco, são apresentados os resultados dos testes de hipótese e da análise dos dados. Na última seção, por fim, são feitas as considerações finais deste estudo.

\section{BREVE CONTEXTUALIZAÇÃO DO LUXO NO CENÁRIO ECONÔMICO ATUAL E DEŚENVOLVIMENTO DAS HIPÓ- TESES DE PESQUISA}

O universo do luxo atrai muitos consumidores, mesmo com a recorrência de crises econômicas. Estima-se que o mercado global de bens de luxo movimente valores próximos a $€ 1$ trilhão. Desse total, as categorias classificadas como tradicionais respondem pela menor parte, aproximadamente $€$ 280 bilhões. Relógios e joias correspondem a $€ 100$ bilhões; moda e vestuário a $€ 50$ bilhões; artefatos e acessórios de couro a $€ 50$ bilhões; bebidas alcoólicas e alimentos a $€ 50$ bilhões; e cosméticos e perfumes a $€ 30$ bilhões. As novas categorias são representadas por turismo e hotéis( $€ 270$ bilhões), tecnologia ( $€ 100$ bilhões), móveis e decoração ( $€ 40$ bilhões), spas, clubes e outros estabelecimentos ( $€ 20$ bilhões) e automóveis ( $€ 250$ bilhões) (BELLAICHE; MEI-POCHTLER; HANISCH, 2010). 
A indústria de bens de luxo parece ter minimizado as perdas causadas pela grande recessão de modo mais rápido que o esperado. Entretanto, a crise resultou em um ponto de inflexão para várias tendências observadas no setor, principalmente com relação às mudanças no comportamento de consumidores e no panorama competitivo. O conceito icônico e de exclusividade não foram mais suficientes para proporcionar um crescimento sustentado das marcas. Como consequência, cada vez menos, os consumidores estavam dispostos a aceitar apenas preços altos como sinônimos de luxo. Mercados tradicionais como a Europa e os Estados Unidos chegaram a um ponto de saturação que obrigou as empresas a se adaptarem a essa nova realidade (BELLAICHE; MEI-POCHTLER; HANISCH, 2010).

O cenário de constante e forte crescimento econômico e a rápida superação dos efeitos gerados pela crise de 2008 transformaram países emergentes, como Brasil, Rússia, Índia e China, nas principais alternativas para a expansão de mercado. Essa tendência, que já ocorria antes da crise, ganhou força a partir de então e possibilitou a expansão das fronteiras das empresas do setor de luxo para os mercados de massa, os quais concentram as maiores taxas de crescimento de milionários no mundo - indivíduos com ativos superiores a US\$ 1 milhão (BELLAICHE; MEI-POCHTLER; HANISCH, 2010; CAPGEMINI; MERRILL LYNCH GLOBAL WEALTH MANAGEMENT, 2011). Fundamentalmente, o que está por trás do crescimento desse mercado é o aumento do consumo de produtos que, via de regra, não fazem parte da cesta de consumo básica de um típico consumidor. Esse aumento seria, portanto, impulsionado por consumidores de maior poder aquisitivo que estariam dispostos a pagar preços mais altos por bens considerados não essenciais, ainda mais tendo em vista a diminuição da desigualdade na distribuição do consumo em países como o Brasil (NETO; MENEZES, 2010).

Embora o país esteja em uma fase quase embrionária no que se refere ao setor de luxo, as importações desse gênero de bens cresceram aproximadamente cinco vezes na última década, passando de US\$2,4 bilhões em 2000 para mais de US\$ 12 bilhões em 2010. Enquanto que no ano de 2000 o volume de bens de luxo importados correspondia a pouco mais de $4 \%$ do montante importado, em 2010 essa relação aumentou para quase 10\%. A figura 1, exposta a seguir, indica que desde 2005 as importações de bens de luxo apresentam um crescimento significativo. Em meados de 2008, com a iminência da crise financeira, as importações foram abruptamente reduzidas, mas retomaram o seu ritmo de crescimento após os choques iniciais gerados pela crise. Considerando-se o período entre janeiro de 2005 e janeiro de 2010, é possível afirmar que as importações têm crescido a uma taxa média superior a 33\% ao ano (BRASIL, 2010).

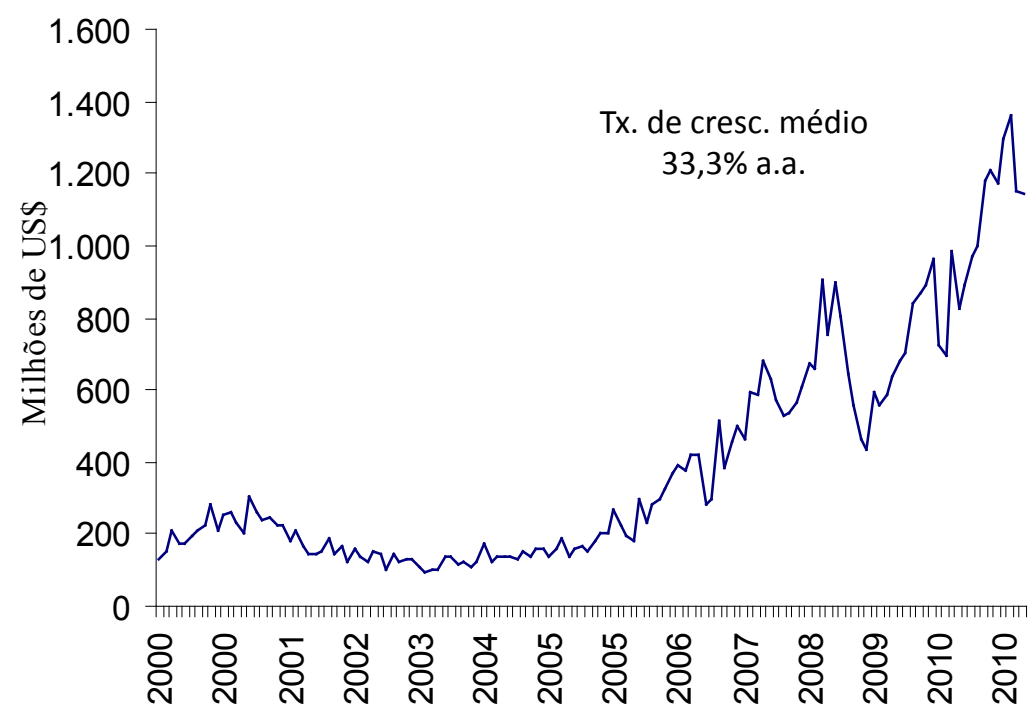

Figura 1. Volume de bens de luxo importados entre janeiro de 2000 e fevereiro de 2011 (milhões de US\$\$/mês)Fonte: Secretaria de Comércio Exterior (SECEX). Sistema de Análise das Informações de Comércio Exterior via Internet. 
Duas causas podem estar diretamente relacionadas ao crescimento das importações. A primeira é o aumento da renda. Conforme demonstrado na figura 2 , logo após a apresentação do Plano Real, a economia brasileira passou por um período de rápido crescimento de seu produto, originando uma tendência que foi interrompida principalmente em decorrência de fatores externos e de incertezas geradas, em 2002, pela possibilidade de eleição de um candidato de esquerda para a presidência da república. Mesmo sob essas condições, o período compreendido entre 1994 e 2002 teve uma taxa de crescimento médio de 0,89\% ao ano. Superadas as incertezas quanto ao novo governo, observou-se uma mudança significativa na tendência de crescimento do produto: a taxa de crescimento médio aumentou para $5,54 \%$ ao ano, o que resultou em um aumento de $45,87 \%$ no período. Se considerado o período total entre 1994 e 2010, verifica-se que o PIB brasileiro cresceu aproximadamente $45 \%$ em termos reais (INSTITUTO BRASILEIRO DE GEOGRAFIA E ESTATÍSTICA, 2010).

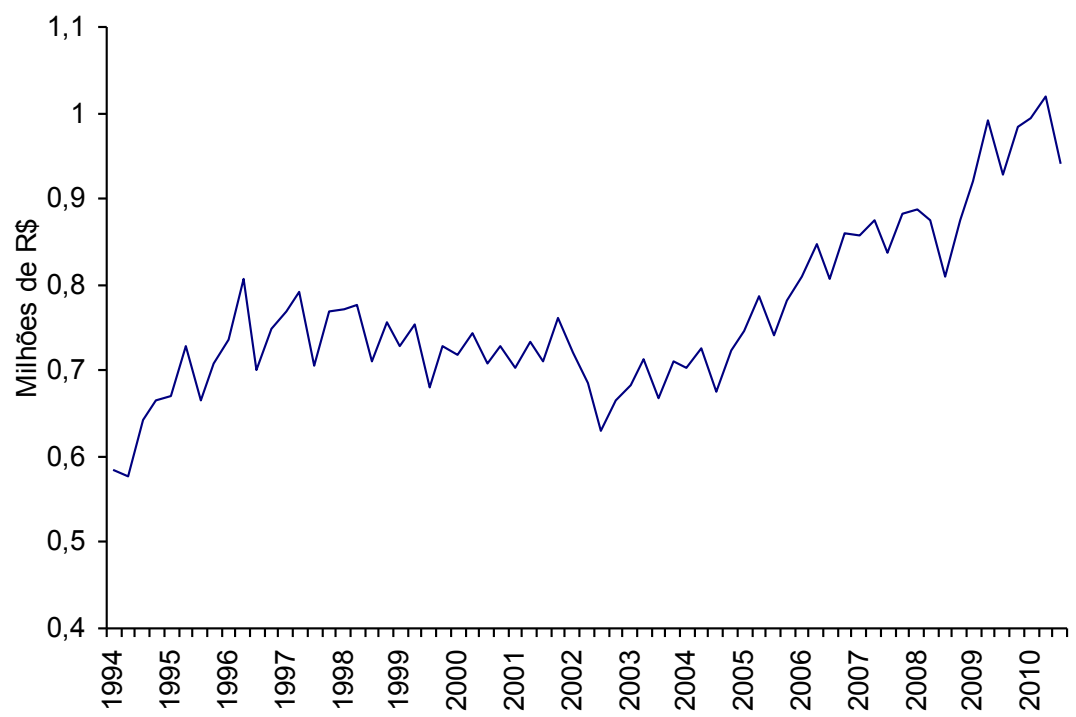

Figura 2. Produto Interno Bruto (PIB) trimestral a preços de mercado (valores reais, deflator: IGP-DI, preços de 2011) Fonte: Sistema de Contas Nacionais do Instituto Brasileiro de Geografia e Estatística (IBGE)

O fenômeno de crescimento da renda também é observado quando são analisados resultados da Pesquisa Nacional por Amostra de Domicílios (PNAD). Entre 2000 e 2009, o rendimento médio mensal do brasileiro aumentou, em termos reais, 16,3\%. Em regiões como nordeste, sul e centro-oeste, esses aumentos foram significativamente maiores (31,9\%, $26,6 \%$ e $25,5 \%$, respectivamente). Nesse período, observou-se uma diminuição da desigualdade da distribuição do consumo familiar com cuidados pessoais, habitação, higiene e vestuário, permitindo que houvesse crescimento na renda do cidadão brasileiro e melhora em indicadores sociais como desigualdade, pobreza e bem-estar. Esse avanço foi sustentado, em grande parte, pela expansão da cobertura de programas governamentais de transferência de renda (FERREIRA et al., 2006; NERI, 2006; NETO; MENEZES, 2010).

O segundo fator que pode estar por trás do aumento das importações é a valorização cambial recente. A partir da análise da figura 3, é possível observar que, até 2002 , a taxa de câmbio acumulou significativa desvalorização desde a implantação do Plano Real. Em outubro de 2002 , a taxa de câmbio comercial alcançou valores nominais próximos a R\$/US\$ 4,00. Passadas as incertezas, e com a melhora dos fundamentos macroeconômicos, essa taxa decaiu até agosto de 
2008 , quando seu valor nominal chegou a $\mathrm{R} \$ 1,55$, indicando uma queda de aproximadamente $60 \%$ no valor da moeda norte-americana. Essa tendência foi subitamente interrompida com a crise financeira de 2008 e prosseguiu em ritmo de queda até o fim do período analisado.

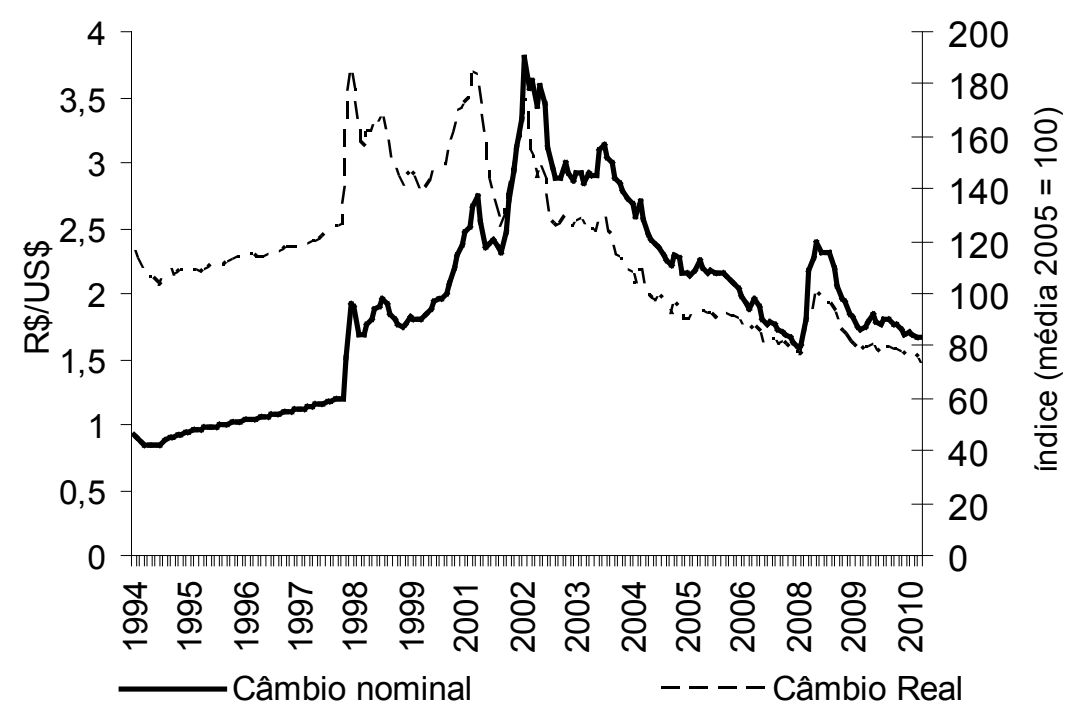

Figura 3. Taxa de câmbio comercial nominal (R\$/US\$) e efetiva real Fonte: Instituto de Pesquisa Econômica Aplicada (IPEA). IPEADATA.

A valorização da moeda nacional pode ter impactos diretos sobre as importações de bens de luxo. A valorização cambial provoca dois efeitos sobre a demanda de produtos importados: em primeiro lugar, torna os preços de mercadorias importadas mais baratos; em segundo, implica maior poder aquisitivo aos brasileiros com relação à compra dos importados. Tendo em vista que o crescimento das importações é observado a partir de 2004, esse fenômeno pode estar diretamente relacionado à valorização cambial observada desde então. Nesse sentido, compreender os determinantes da demanda por bens de luxo é tarefa importante, uma vez que o Brasil faz parte do grupo de países emergentes e figura como um dos alvos das estratégias de expansão de diversas empresas que compõem o mercado de bens e serviços de luxo. $O$ crescimento das importações brasileiras desse gênero de produtos parece ser determinado, a priori, pelo aumento da renda e também pela valorização cambial, pressuposições que levam à construção das seguintes afirmativas sobre os parâmetros populacionais (LARSON. FARBER, 2010; TRIOLA, 2005):

Hipótese 1: aumentos no preço (taxa de câmbio real) proporcionam variações negativas na demanda por bens de luxo (volume de importações de produtos da NCM).

Hipótese 2: aumentos na renda doméstica (PIB) proporcionam variações positivas na demanda por bens de luxo (volume de importações de produtos da NCM). 


\section{MÉTODO E PROCEDIMENTOS}

Para a análise dos determinantes do consumo de bens de luxo, utilizou-se um modelo de demanda por importações, segundo o qual as importações são representadas pela seguinte função:

$$
I M=I M(Y, \varepsilon), \quad I M_{Y}>0 \text { e } I M_{f}<0
$$

onde $I M$ é a quantidade importada, $Y$ é a renda doméstica e $\varepsilon$ é a taxa de câmbio real. A taxa de câmbio real, por sua vez, é definida como $\varepsilon \equiv E P * / P^{\prime}$, onde $E$ é a taxa câmbio nominal, $P^{*}$ é o nível de preço do estrangeiro e $P$ é o nível de preços interno. As relações esperadas, a priori, são que aumentos na renda doméstica proporcionem aumento na demanda por importações, enquanto que aumentos na taxa de câmbio real (desvalorização cambial) provoquem queda na demanda por importações. A taxa de câmbio real demonstra que, quanto mais alto o preço dos bens externos em relação ao preço dos bens internos, menor será a demanda interna por bens externos.

A versão empírica da função de demanda por importações (eq. (1)) baseia-se no modelo de Thirlwall (2002) e é especificada como uma função multiplicativa de seus argumentos - preços das importações, preços dos bens domésticos e renda doméstica -, podendo ser representada como:

$$
I M_{t}=\left(\frac{E_{i} P_{i}^{*}}{P_{i}}\right)^{\beta_{1}} Y_{t}^{\beta_{2}}=\varepsilon_{t}^{\beta_{1}} Y_{i}^{\beta_{1}},
$$

onde as variáveis são definidas como anteriormente e $b_{1}$ e $b_{2}$ são os parâmetros a serem estimados. Tomando o logaritmo natural de ambos os lados da equação (2), tem-se que as importações podem ser determinadas na forma linear como:

$$
\ln M_{\mathrm{i}}=\beta_{0}+\beta_{\mathbb{1}} \ln \varepsilon_{\mathrm{i}}+\beta_{\mathrm{z}} \ln Y_{\mathrm{i}}+u_{\mathrm{i}}
$$

onde os sinais esperados são: $\beta_{1}<0$ e $\beta_{2}>0$. A função empírica da forma como é representada corresponde a uma forma funcional log-log e uma de suas características é que os coeficientes de inclinação representam as elasticidades, diretamente. Assim, $\beta_{1}$ corresponde à elasticidade-preço da demanda das importações e $\beta_{2}$ corresponde à elasticidade-renda (GUJARATI; PORTER, 2011).

O modelo empírico (eq. (3)), por ser tratar de uma função linear nos parâmetros, permite que seja utilizado o método de Mínimos Quadrados Ordinários (MQO) para estimar os parâmetros. Entretanto, por se tratar de um modelo que utiliza séries de dados temporais, torna-se fundamental testar a hipótese de estacionariedade das séries empregadas. Caso as séries não sejam estacionárias, os resultados da regressão podem não ser válidos, uma vez que pode haver um problema de regressão espúria. Em tais casos, os testes $t$ e $F$ usuais não serão válidos, e, consequentemente, testes de hipóteses não poderão ser feitos com base nas estimativas encontradas (GUJARATI; PORTER, 2011).

Mesmo na presença de não estacionariedade das séries, as estimativas obtidas ainda podem ser válidas caso as variáveis utilizadas sejam cointegradas. Duas ou mais séries estão cointegradas se as mesmas se movem conjuntamente no tempo e suas diferenças forem são estáveis (estacionárias), mesmo que cada série em particular tenha uma tendência estocástica e seja, portanto, não estacionária. A cointegração reflete a presença de um equilíbrio de longo prazo para o qual o sistema econômico converge. Segundo Gujarati e Porter (2011), se cada série é um processo não estacionário e integrado de ordem um, a possibilidade de equilíbrio pode ser testada utilizando o teste de cointegração de Engle-Granger (EG). 
O teste de EG consiste em testar a estacionariedade dos resíduos da equação (3). Caso os resíduos dessa equação sejam estacionários, tem-se que as variáveis $I M, \varepsilon$ e $Y$ são cointegradas e possuem, portanto, uma relação de longo prazo. Especificamente em casos nos quais o número de variáveis é maior que dois, aconselha-se utilizar o teste de Johansen (1991). Entretanto, por questões de simplicidade, optou-se por manter a abordagem proposta pelo teste EG. O fato de as variáveis $I M, \varepsilon$ e $Y$ possuírem uma relação de longo prazo não implica que no curto prazo as variáveis estarão em equilíbrio. Dessa forma, utilizam-se os resíduos da equação original (3) como uma forma de interligar o comportamento de curto prazo das importações (IM) e seu valor de longo prazo. Esse método é conhecido como Mecanismo de Correção de Erro (MCE) e é representado pela seguinte equação:

$$
\Delta \ln I M_{t}=\alpha_{0}+\alpha_{1} \Delta \ln \varepsilon_{t}+\alpha_{2} \Delta \ln Y_{t}+\alpha_{3} \hat{u}_{i-1}+v_{t}
$$

onde $\Delta$ denota o operador de primeira diferença, $v_{t}$ é o termo de erro aleatório e $\hat{u}_{t-1}=\ln I M_{t-1}-\beta_{0}-\beta_{1} \ln \varepsilon_{i-1}-\beta_{2} \ln Y_{i-1}$, isto é, o valor defasado em um período do erro da regressão cointegrante. A equação do mecanismo de correção de erro (4) mostra que $\Delta / n / M$ depende de $\Delta / n \varepsilon$, de $\Delta / n Y$ e também do termo de erro de equilíbrio.

\section{VARIÂVEIS UTILIZADAS}

Produtos de tecnologia, joias, relógios, perfumes, veículos, vestuários e os classificados como novas categorias, relacionados a experiências e serviços, podem corresponder ou não ao mercado de luxo. Classificá-los depende de suas características intrínsecas. Nesse sentido, reunir todos os bens e serviços considerados de luxo em um único índice representaria uma tarefa difícil de ser realizada, até mesmo porque a literatura parece ser omissa na definição conceitual de um produto de luxo. Segundo Roper et al. (2013), há uma dificuldade prática em identificar a essência caracterizadora de algo luxuoso. Pressupondo essa dificuldade, um levantamento promovido na literatura da área de marketing permitiu a definição de categorias que provavelmente corresponderiam ao gênero de luxo. Esse procedimento permitiu uma etapa posterior da abordagem metodológica, que foi a definição de mais de mil itens da tabela de produtos da NCM de importações brasileiras. De acordo com Pianaro e Marcondes (2010), a maioria dos bens de luxo tradicionais no Brasil pode ser considerada importada, uma vez que normalmente se caracteriza por possuir marca reconhecida em nível mundial.

Desde janeiro de 1995, os países do Mercosul adotam a tabela NCM, que tem por base o Sistema Harmonizado de Designação e de Codificação de Mercadorias ou Sistema Harmonizado (SH). Esse método internacional de classificação de mercadorias, baseado em uma estrutura de códigos e suas respectivas descrições, ajuda a promover o desenvolvimento do comércio internacional, da coleta de dados, das análises estatísticas, da elaboração de tarifas etc. O código SH é formado por seis dígitos, que mostram as especificidades do produto, tais como origem, matéria constitutiva e aplicação, em um ordenamento numérico crescente e de acordo com o nível de sofisticação das mercadorias. Assim, dos oito dígitos que compõem a NCM, os seis primeiros são formados pelo $\mathrm{SH}$, enquanto que o sétimo e oitavo dígitos correspondem a desdobramentos específicos atribuídos no âmbito do Mercosul (BRASIL, 2011). 
A partir da tabela NCM, foi determinada uma lista com aproximadamente mil produtos importados, considerados, segundo comparação com a literatura, bens de luxo. No sistema de classificação, esses produtos estão distribuídos ao longo de 20 capítulos e 89 subcapítulos. Em alguns casos, os produtos correspondem a um capítulo na íntegra, como, por exemplo: "Cap. 71 - pérolas naturais ou cultivadas, pedras preciosas". Em outros, a um produto específico, como por exemplo: "Código NCM no. 8711.50 .00 - motocicletas, etc. c/ motor pistão alternat. Cil.>$\left.800 \mathrm{~cm}^{3}\right)$ ". A tabela A, na seção Apêndice, resume capítulos e produtos selecionados da Tabela NCM para a operacionalização dessa pesquisa.

A série temporal foi construída a partir da soma das importações desses produtos, obtida junto ao Ministério do Desenvolvimento, Indústria e Comércio Exterior (MDIC) no sistema AliceWeb, e corresponde ao volume de importações (em US\$) entre janeiro de 2000 e fevereiro de 2011 (série representada na Figura 1). Como os preços dos produtos importados não estão disponíveis, foi necessário o uso de uma proxy. Desse modo, optou-se pela taxa de câmbio efetiva real das exportações de produtos manufaturados (série representada na Figura 3). Segundo o IPEA, este índice é calculado como média ponderada do índice de paridade do poder de compra dos 16 maiores parceiros comerciais do Brasil e representa a competitividade das exportações brasileiras. Por sua vez, a paridade do poder de compra é definida pelo quociente da taxa de câmbio nominal (em R\$়/US\$) e pela relação entre o Índice de Preço por Atacado (IPA) do país estrangeiro e o Índice de Preços por Atacado da indústria de transformação (IPA-IT/FGV) do Brasil. As ponderações utilizadas foram as participações de cada parceiro no total das exportações brasileiras de manufaturados em 2001 (INSTITUTO DE PESQUISA ECONÔMICA APLICADA, 2011).

A taxa de câmbio real $(\varepsilon)$ foi obtida no banco de dados do IPEA e corresponde à variável taxa de câmbio efetiva real (IPA-IT) - exportações - manufaturados - índice (média em 2005 = 100). Por fim, uma vez que as séries anteriores tinham periodicidade mensal, optou-se por utilizar como proxy da renda doméstica $(Y)$ a série mensal do PIB, calculada pelo Banco Central do Brasil (BCB) e obtida no seu banco de dados.

\section{DISCUSSÃO DOS RESULTADOS}

Como primeira etapa da análise, foi testada a hipótese de estacionariedade das séries utilizadas. Conforme destacado na seção anterior, as estimativas de MQO serão válidas apenas na presença de séries temporais estacionárias. Caso contrário, torna-se necessário testar também a hipótese de cointegração para determinar a validade do modelo estimado. $O$ teste utilizado foi o teste de Dickey-Fuller Aumentado (ADF), cuja hipótese nula é existência de raiz unitária na série (GUJARATI; PORTER, 2011).

$O$ teste $A D F$ foi aplicado às três séries sob três formas funcionais alternativas: com constante e tendência; constante; e sem constante e sem tendência. De acordo com os resultados, resumidos na tabela 1 , as três séries mostraram-se não estacionárias em nível, uma vez que todos os testes não foram estatisticamente significativos, ao nível de $5 \%$ de significância. $O$ teste das mesmas variáveis, porém, em primeira diferença, rejeitou a hipótese nula de raiz unitária, permitindo concluir, portanto, que as séries utilizadas na pesquisa são não estacionárias em nível, mas estacionárias em primeira diferença. Ou seja, é possível afirmar que todas as variáveis são integradas de ordem um. 
Teste de raiz unitária de Dickey-Fuller Aumentado (ADF)

\begin{tabular}{cccc|ccc}
\hline & \multicolumn{3}{c|}{ Variável em nível } & \multicolumn{2}{c}{ Variável em primeira diferença } \\
\cline { 2 - 6 } & $\begin{array}{c}\text { Com const. e } \\
\text { tendência }\end{array}$ & Constante & $\begin{array}{c}\text { Sem constante e } \\
\text { sem tendência }\end{array}$ & $\begin{array}{c}\text { Com const. e e } \\
\text { tendência }\end{array}$ & Constante $\begin{array}{c}\text { Sem constante e } \\
\text { sem tendência }\end{array}$ \\
\hline$I M$ & $-2,496$ & 0,417 & 1,143 & $-2,490$ & $-1,888$ & $-1,650^{* *}$ \\
$\varepsilon$ & $-2,620$ & $-0,288$ & $-1,789 *$ & $-6,147 * * *$ & $-6,151^{* * *}$ & $-5,837^{* * *}$ \\
$Y$ & $-2,082$ & $-0,760$ & 4,075 & $-4,419 * * *$ & $-4,390 * * *$ & $-1,299$ \\
\hline
\end{tabular}

Fonte: Resultados da pesquisa

Nota: ${ }^{* * *}$ significativo a $1 \%,{ }^{* *}$ significativo a $5 \%,{ }^{*}$ significativo a $10 \%$.

Estatísticas tau $(\tau):\left(\tau_{\tau}\right)$ tendência e constante, $\left(\tau_{\mu}\right)$ apenas constante e $(\tau)$ sem tendência e constante

Uma vez que as séries não são estacionárias, tem-se que as estimativas do modelo só serão válidas sob a hipótese de cointegração. A tabela 2 , exposta a seguir, resume os resultados da estimação do modelo de demanda por importações de longo prazo (eq. (3)). O resultado do teste de cointegração de EG (Teste Tau), realizado nos resíduos do modelo estimado, foi igual a $-3,607$ com $p$-valor igual a 0,0791 . Se for considerado um nível de significância de $10 \%$, o resultado permite rejeitar a hipótese nula de que as séries não são cointegradas e, portanto, possuem uma relação de longo prazo. Esse resultado também possibilita concluir que as estimativas obtidas são não enviesadas e que a realização dos testes de hipóteses são válidas.

Tabela 2:

Resultados da relação de longo prazo entre $l M, \varepsilon$ e $Y$

\begin{tabular}{cccc}
\hline Var. dependente & Constante & $\ln \varepsilon$ & $\ln Y$ \\
\hline $\ln I M$ & 5,007 & $-0,428$ & $1,365^{* * *}$ \\
& $(5,263)$ & $(0,403)$ & $(0,286)$ \\
R - quadrado & 0,669 & $\mathrm{~F}(2,131)$ & 132,64 \\
$\mathrm{R}$ - quad. ajustado & 0,664 & $\mathrm{P}-\mathrm{valor}(\mathrm{F})$ & 0,0000 \\
Critério de Akaike & 159,57 & Critério de Schwarz & 168,27 \\
Durbin-Watson & 0,1441 & & \\
\hline
\end{tabular}

Fonte: Resultados da pesquisa

Nota: ${ }^{* *}$ significativo a $1 \%, * *$ significativo a $5 \%, *$ significativo a $10 \%$.

De acordo com os resultados da tabela 2, verifica-se que o modelo se ajusta muito bem aos dados, uma vez que o coeficiente $R^{2}$ obtido foi igual a 0,66 , o que representa uma parcela significativa da variação das importações explicada apenas pelas duas variáveis do modelo. Além disso, observa-se que os sinais obtidos estão de acordo com o esperado a priori e que os parâmetros são, com base no teste $F$, conjuntamente, estatisticamente significativos. Com relação à significância estatística individual, entretanto, verifica-se que apenas a variável renda se mostrou estatisticamente diferente de zero, indicando que, na função de demanda por importações, segundo os resultados encontrados, apenas a variável renda exerce influência sobre a demanda de bens de luxo. Os resultados permitem verificar que um aumento de $1 \%$ na renda implica um aumento de $1,36 \%$ na demanda, resultado que encontra respaldo na teria econômica, que classifica bens de luxo apenas por sua elasticidade-renda da demanda superior a um (KEMP, 1998; VARIAN, 2009). 
Dando prosseguimento à análise, uma vez que as variáveis do modelo são cointegradas, ou seja, há uma relação de equilíbrio de longo prazo entre elas, um modelo de curto prazo, representado pelo MCE, também pode ser estimado. O modelo MCE (eq. (4)) corrige os desequilíbrios e fornece estimativas das relações de curto prazo entre as variáveis. A tabela 3 resume, assim, os resultados da estimação da equação de curto prazo e relaciona a variação nas importações de bens de luxo com a variação na renda e na taxa de câmbio real e com o termo de erro de equilíbrio, defasado em um período. Nessa regressão, $\Delta \ln \varepsilon$ e $\Delta \ln Y$ capturam as perturbações em $Y$ e $\varepsilon$, respectivamente, enquanto que o termo de correção de erro captura o ajustamento para o equilíbrio de longo prazo.

Tabela 3:

Resultados do modelo MCE

\begin{tabular}{ccccc}
\hline Var. dependente & Constante & $\ln \varepsilon$ & $\Delta \ln Y$ & $\hat{u}_{t-1}$ \\
\hline$\Delta \ln I M$ & $-0,001$ & 0,019 & $1,892^{* * *}$ & $-0,075^{* *}$ \\
& $(0,014)$ & $(0,406)$ & $(0,380)$ & $(0,032)$ \\
$R-$ quadrado & 0,189 & $\mathrm{~F}(2,131)$ & 10,079 & \\
$\mathrm{R}-$ quad. ajustado & 0,171 & P-valor(F) & 0,0000 & \\
Critério de Akaike & $-104,23$ & Critério de Schwarz & $-92,67$ & \\
Durbin-Watson & 2,534 & & & \\
\hline
\end{tabular}

Fonte: Resultados da pesquisa

Nota: ${ }^{* *}$ significativo a $1 \%,{ }^{* *}$ significativo a $5 \%, *$ significativo a $10 \%$.

De acordo com os resultados obtidos, novamente a variável taxa de câmbio real não é estatisticamente significativa, nem ao nível de $10 \%$ de significância. A variável renda, por sua vez, é estatisticamente significativa, ao nível de $1 \%$, o que indica que alterações no curto prazo na renda têm efeitos positivo sobre as importações de bens de luxo, ou seja, uma variação de $1 \%$ na variável $\Delta \ln Y$ resulta em uma variação em $\Delta \ln / M$ de 1,89\%, no curto prazo, correspondendo a uma elasticidade maior do que a encontrada em longo prazo. Outro ponto importante é a significância estatística do termo de erro, que nesse caso é significativo ao nível de $5 \%$. Isso demonstra que cerca de $7 \%$ da discrepância entre o valor efetivo e o valor de longo prazo ou de equilíbrio de importações de bens de luxo é eliminada ou corrigida a cada mês.

A não significância estatística da variável taxa de câmbio (que representa a variável preço do modelo) pode estar relacionada a dois fatores. O primeiro diz respeito a uma limitação metodológica, no que diz respeito a proxy utilizada. Como não existem informações sobre o preço dos produtos importados, utilizou-se a taxa de câmbio real de produtos manufaturados. Essa taxa leva em consideração um índice de preço externo e interno com base em uma cesta de produtos manufaturados, não necessariamente os mesmos produtos importados considerados no estudo. Desse modo, os preços representados pelos índices $P^{*}$ e $P$ podem não refletir corretamente os preços dos bens analisados. Em segundo lugar, a não significância estatística pode ser decorrência da própria característica da demanda por bens de luxo. Esses resultados estão de acordo com a discussão promovida por Leibenstein (1950) sobre demanda não funcional, mais especificamente o que o autor classifica como efeito esnobe: quando o consumidor atribui utilidade em função do caráter exclusivo do produto e não do seu preço. $O$ fator exclusividade estaria relacionado, ainda, à argumentação proposta por Sá e Marcondes (2010) a respeito dos objetos de luxo: estes são acrescidos de sensações, conceitos e imagens. Por essas razões, o consumidor 
estaria disposto a adquiri-lo mesmo diante de um preço mais elevado. Tais considerações foram corroboradas pelos resultados provenientes do modelo estimado, no qual variações nos preços não provocaram variações estatisticamente significativas na quantidade da demanda.

\section{CONSIDERAÇÕES FINAIS}

O mercado de bens de luxo possui uma característica que o distingue de forma importante de outros mercados da economia: o perfil dos seus consumidores. Uma vez que esse mercado é movido por sensações e desejos que extrapolam as necessidades básicas de um consumidor típico, a sua demanda acaba sendo definida por padrões que, normalmente, contrariam os pressupostos da teoria econômica. Os consumidores do luxo parecem ser guiados por uma motivação emocional relacionada a um prazer que esses objetos oferecem, de modo que, seja pelo status ou pela distinção social, o desejo é diferenciar-se das outras pessoas ou afirmar-se em determinados grupos sociais.

O presente trabalho teve como objetivo analisar os determinantes da demanda por bens de luxo no Brasil por meio de uma abordagem alternativa, fazendo uso de um modelo de demanda por importações. Foi possível verificar que o fator determinante das importações no período pesquisado foi a renda, enquanto que o preço, representado pela taxa de câmbio real, mostrou-se estatisticamente não significativo. Esse resultado, conforme discutido, pode ser consequência de uma limitação metodológica, uma vez que se utilizou como proxy de preços a taxa de câmbio real, ou pode ser resultado das características da própria demanda, o que exige investigações adicionais. As elasticidades-renda das demandas estimadas foram 1,36 no longo prazo e 1,892 no curto prazo. Esses resultados foram estatisticamente significativos e estão de acordo com a teoria econômica, que classifica os bens de luxo apenas por sua elasticidade-renda da demanda superior a um. Em último caso, isso implica que aumentos da renda provocam impactos positivos e mais que proporcionais na quantidade demandada por bens e serviços do gênero de luxo.

\section{REFERÊNCIAS}

ALLÉRÈS, D. Luxo... estratégias, marketing. Tradução: Mauro Gama. Rio de Janeiro: FGV, 2000.

BELLAICHE, J.-M.; MEI-POCHTLER, A.; HANISCH, D. The new world of luxury: caught between growing momentum and lasting change. The Boston Consulting Group, dez. 2010. Disponível em: <http://www.bcg.com/ documents/file67444.pdf>. Acesso em: 21 out. 2011

BRASIL. Secretaria de Comércio Exterior (SECEX), do Ministério do Desenvolvimento, Indústria e Comércio Exterior (MDIC). Sistema de Análise das Informações de Comércio Exterior via Internet. Brasília, 2010. Disponível em: <http://aliceweb.desenvolvimento.gov. br>. Acesso em: 14 jun. 2009.

BRASIL. Conselho de Ministros da Câmara de Comércio Exterior (CAMEX). Resolução no 94, de 8 de dezembro de 2011. Altera a Nomenclatura Comum do Mercosul - NCM e a Tarifa Externa Comum - TEC, a Lista de Exceções à TEC e a Lista de Exceções de Bens de Informática e Telecomunicações - BIT para adaptação às modificações do Sistema Harmonizado (SH2012). Disponível em: <www.desenvolvimento. gov.br/arquivos/dwnl_1323716926.pdf>. Acesso em: 21 out. 2011.

BRUNELLI, $M$. et al. Shopping experience no mercado de luxo: caso $H$. Stern. Revista Pensamento Contemporâneo em Administração, v. 6, n. 2, p. 14-32, abr./jun. 2012. 
CAPGEMINI; MERRILL LYNCH GLOBAL WEALTH MANAGEMENT. 2011 World Wealth Report. [S.I.], 2011. Disponível em: <http://www. $\mathrm{ml} . c 0 \mathrm{~m} / \mathrm{media} / 114235 . \mathrm{pdf}>$. Acesso em: 21 out. 2011.

CAROZZI, F. Mercado de Luxo no Mundo e no Brasil e Christian Dior: reposicionamento de marca no setor de luxo. Revista da ESPM, v.12, n.1, p.110-115, jan./fev. 2005.

CASTARÉDE, J. O Luxo: os segredos dos produtos mais desejados do mundo. São Paulo. Barcarolla, 2005.

DUBOIS, B.; DUQUESNE, P. The market for luxury goods: income versus culture. European Journal of Marketing, [S.I.], v. 27, n. 1, p. 3544, 1993.

FERREIRA, F. et al. Ascensão e queda da desigualdade de renda no Brasil. Econômica, $v$. 8, n. 1, p. 147-169, jun. 2006.

FERREIRA, J. B. et al. O mercado de luxo do Rio de Janeiro: uma análise da percepção das consumidoras cariocas. Revista ADM. MADE, v. 17, n. 1, p. 66-84, mai./ago. 2013.

FIGUEIREDO, E.; SILVA, C. Desigualdade de oportunidades no Brasil: uma decomposição quantílica contrafactual. Pesquisa e Planejamento Econômico, v. 42, n. 1, p. 41-60, abr. 2012.

FINESTRALLI, M.; GARRIDO, I. O uso de referências da identidade cultural brasileira no marketing internacional de produtos e marcas de luxo. In: ENCONTRO DA ASSOCIAÇÃO NACIONAL DE PÓS-GRADUAÇÃO E PESQUISA EM ADMINISTRAÇÃO, XXXII., 2008, Rio de Janeiro. Anais... Rio de Janeiro, 2008, CD-ROM.

GIACALONE, J. The market for luxury goods: the case of the Comite Colbert. Southern Business Review, Statesboro, v. 32, n. 1, p. 3340, Fall. 2006.

GROTH, J. The exclusive value principle - a concept for marketing. Journal of Product \&
Brand Management, v. 3, n. 3, p. 8-18, 1994.

GUJARATI, D.; PORTER, D. Econometria básica. 5. ed. Porto Alegre: AMGH, 2011.

GUPTA, K. Changing paradigms of luxury consumption in India: a conceptual model. South Asian Journal of Management, v. 16, n. 4, p. 29-43, out./dez. 2009.

HUDDERS, L. Why the devil wears Prada: consumers' purchase motives for luxuries. Journal of Brand Management, v. 19, p. 609622, 2012.

HUSIC, M.; CICIC, M. Luxury consumption factors. Journal of Fashion Marketing and Management, [S.I.], v. 13, n. 2, p. 231-245, 2009.

INSTITUTO BRASILEIRO DE GEOGRAFIA E ESTATÍSTICA (IBGE). Sistema de Contas Nacionais. Brasília, 2010. Disponível em: <http://www.ibge.gov.br>. Acesso em: 14 jun. 2009.

INSTITUTO DE PESQUISA ECONÔMICA APLICADA (IPEA). IPEADATA. Séries temporais históricas. Brasília, 2011. Disponível em: <http://www.ipeadata.gov.br>. Acesso em: 15 mai. 2011.

JOHANSEN, S. Estimation and hypothesis testing of cointegration vectors in Gaussian vector autoregressive models. Econometrica. V. 59, p. 1551-1580, 1991.

KAPFERER, J. Why should luxury should not delocalize - a critique of a growing tendency. The European Business Review, p. 58-62, mar./abr. 2012.

KEMP, S. Perceiving luxury and necessity. Journal of Economic Psychology, v. 19, p. 591606, 1998.

LARSON, R.; FARBER, B. Estatística aplicada. Tradução: Luciane Ferreira Pauleti Vianna. 4. ed. São Paulo: Pearson Prentice Hall, 2010.

LEIBENSTEIN, H. Bandwagon, snob, and Veblen 
effects in the theory of consumers' demand. The Quarterly Journal of Economics, v. 64, n. 2, p. 183-207, mai. 1950.

MOUSINHO, M.; PEREIRA, R.; STORNI, M. Usando o luxo, consumindo o espaço: uma investigação sobre as relações entre espaço, consumo de moda e luxo. In: ENCONTRO DE MARKETING DA ASSOCIAÇÃO NACIONAL DE PÓS-GRADUAÇÃO E PESQUISA EM ADMINISTRAÇÃO, IV., 2010, Florianópolis. Anais... Rio de Janeiro, 2010, CD-ROM.

NERI, M. Desigualdade, estabilidade e bemestar social. Ensaios Econômicos, Rio de Janeiro, n. 637, dez. 2006. Disponível em: <http://bibliotecadigital.fgv.br/dspace/ bitstream/handle/10438/811/2168. pdf?sequence=1>. Acesso em: 21 out. 2011.

NETO, R.; MENEZES, T. Nível e evolução da desigualdade dos gastos familiares no Brasil: uma análise para as regiões metropolitanas no período de 1996 a 2003. Estudos Econômicos, v. 40, n. 2, p. 341-372, abr./jun. 2010.

PEREIRA, L.; BIDO, D.; KIMURA, H. Consumo de luxo no Brasil - um estudo sobre a demanda. FACEF Pesquisa, v. 13, n. 3, p. 325-342, 2010.

PIANARO, L.; MARCONDES, R. Identificação de conceitos, significado e atributos dos produtos de luxo no mercado de roupas e acessórios. Revista Alcance Eletrônica, v. 17, n. 1, p. 7383, jan./mar. 2010.

PINTO, M. R.; LARA, J. E. O que se publica sobre comportamento do consumidor no Brasil, afinal? Revista de Administração da UFSM, v. 1, n. 3, p. 85-100, set./dez. 2008.

REGO, B. B.; OLIVEIRA, M. O. R.; LUCE, F. B. Uma discussão teórica da relação do valor da marca e do valor do cliente. Revista de Administração da UFSM, v. 1, n. 2, p. 275-290, mai./ago. 2008.

ROPER, S. et al. Constructing luxury brands: exploring the role of consumer discourse. European Journal of Marketing, v. 47, n. 3/4, p. 375-400, 2013.
SÁ, R. L. G., MARCONDES, C. O ponto de vendas de produtos de luxo da moda de vestuário feminino no Brasil. Cadernos EBAPE, v.8, n.3, set. 2010.

SHUKLA, P. The influence of value perceptions on luxury purchase intentions in developed and emerging markets. International Marketing Review, v. 29, n. 6, p. 574-596, 2012.

SHUKLA, P.; PURANI, K. Comparing the importance of luxury value perceptions in cross-national contexts. Journal of Business Research, v. 65, n. 10, out. 2012.

SULEHRI, N. et al. Consumer behavior slide down for purchase of luxury brands in Pakistan; a conceptual framework. European Journal of Economics, Finance and Administrative Sciences, [S.I.], n. 28, p. 189-194, 2011.

THIRLWALL, A. The nature of economic growth: an alternative framework for understanding the performance of nations. Cheltenham: Edward Elgar, 2002.

TRIOLA, M. Introdução à estatística. Tradução: Vera Regina Lima de Farias e Flores. Rio de Janeiro: LTC, 2005.

VARIAN, R. Microeconomia: princípios básicos. 6. ed. São Paulo: Campus, 2009.

VEBLEN, T. The theory of the leisure class. An economic study of institutions. Londres: Macmillan, 1899.

WIEDMANN, K.; HENNIGS, N. Placing luxury marketing on the research agenda not only for the sake of luxury - An introduction. In: WIEDMANN, K.; HENNIGS, N. (Eds.). Luxury marketing: a challenge for theory and practice. New York: Springer, 2013. p. 4-17.

WIEDMANN, K.; HENNIGS, N.; SIEBELS, A. Measuring consumers' luxury value perception: a cross-cultural framework. Academy of Marketing Science Review, [S.I.], v. 11, n. 7, p. 1-21, 2007. 
WIEDMANN, K.; HENNIGS, N.; KLARMANN, C. Luxury consumption in the trade-off between genuine and counterfeit goods: what are the consumers' underlying motives and valuebased drivers? Journal of Brand Management, v. 19, p. 544-566, mar. 2012.

\section{APÊNDICE}

Tabela 4:

Série Temporal de Produtos de Luxo Importados

\begin{tabular}{|c|c|c|}
\hline CAPÍTULO & PRODUTOS & CÓDIGOS \\
\hline 01 - ANIMAIS VIVOS & CAVALOS REPRODUTORES, DE RAÇAA PURA & 0101.11 .00 \\
\hline $\begin{array}{c}03 \text { - PEIXES E CRUSTACEOS, MO- } \\
\text { LUSCOS E OUTROS INVERTEBRA- } \\
\text { DOS AQUÁTICOS }\end{array}$ & & 03 \\
\hline \multirow{7}{*}{$\begin{array}{l}04 \text { - LEITE E LATICÍNIOS, OVOS DE } \\
\text { AVES, MEL NATURAL ETC. }\end{array}$} & $\begin{array}{c}\text { OUTROS. QUEIJOS FRESCOS (NÃO CURADOS), IN- } \\
\text { CLUINDO REQUEIJAO ETC. }\end{array}$ & 0406.10 .90 \\
\hline & $\begin{array}{c}\text { QUEIJOS FUNDIDOS, EXCETO RALADOS OU EM } \\
\text { PÓ }\end{array}$ & 0406.30 .00 \\
\hline & QUEIJOS DE PASTA MOFADA (AZUL) & 0406.40 .00 \\
\hline & $\begin{array}{l}\text { QUEIJOS CONTENDO TEOR DE UMIDADE <36\%, } \\
\text { EM PESO (MASSA DURA) }\end{array}$ & 0406.90 .10 \\
\hline & $\begin{array}{c}\text { QUEIJOS CONTENDO 36\%<= TEOR DE UMIDADE } \\
<46 \%,(\text { MASSA SEMIDURA) }\end{array}$ & 0406.90 .20 \\
\hline & $\begin{array}{c}\text { QUEIJOS CONTENDO } 46 \%<=\text { TEOR DE UMIDADE } \\
<55 \%,(\text { MASSA MACIA) }\end{array}$ & 0406.90 .30 \\
\hline & OUTROS QUEIJOS & 0406.90 .90 \\
\hline \multirow{4}{*}{$\begin{array}{l}15 \text { - GORDURAS,ÓLEOS E CERAS } \\
\text { ANIMAIS OU VEGETAIS ETC. }\end{array}$} & AZEITE DE OLIVA, VIRGEM & 1509.10 .00 \\
\hline & AZEITE DE OLIVA, REFINADO & 1509.90.10 \\
\hline & OUTROS AZEITES DE OLIVA & 1509.90 .90 \\
\hline & $\begin{array}{c}\text { OUTROS ÓLEOS DE AZEITONAS E MISTURAS COM } \\
\text { AZEITES DE OLIVA }\end{array}$ & 1510.00 .00 \\
\hline $\begin{array}{l}16 \text { - PREPARAÇÕES DE CARNE, DE } \\
\text { PEIXES OU DE CRUSTÁCEOS ETC. }\end{array}$ & CAVIAR E SEUS SUCEDÂNEOS & 1604.30 .00 \\
\hline \multirow{2}{*}{$\begin{array}{l}17 \text { - AÇÚCARES E PRODUTOS DE } \\
\text { CONFEITARIA }\end{array}$} & CHOCOLATE BRANCO, SEM CACAU & 1704.90 .10 \\
\hline & $\begin{array}{l}\text { BOMBONS, CARAMELOS, CONFEITOS E PASTI- } \\
\text { LHAS, SEM CACAU }\end{array}$ & 1704.90 .20 \\
\hline \multirow{2}{*}{18 - CACAU E SUAS PREPARAÇÕES } & $\begin{array}{l}\text { CHOCOLATE RECHEADO, EM TABLETES, BARRAS } \\
\text { E PAUS }\end{array}$ & 1806.31 .10 \\
\hline & $\begin{array}{l}\text { CHOCOLATE NÃO RECHEADO, EM TABLETES, BAR- } \\
\text { RAS E PAUS }\end{array}$ & 1806.32 .10 \\
\hline \multirow{2}{*}{$\begin{array}{l}19 \text { - PREPARAÇÕES A BASE DE CE- } \\
\text { REAIS, FARINHAS, AMIDOS ETC. }\end{array}$} & $\begin{array}{l}\text { MASSAS ALIMENTÍCIAS, CONTENDO OVOS, NÃO } \\
\text { COZIDAS, NÃO RECHEADAS ETC. }\end{array}$ & 1902.11 .00 \\
\hline & $\begin{array}{l}\text { MASSAS ALIMENTÍCIAS RECHEADAS, INCLUINDO } \\
\text { COZIDAS, PREPARAÇÕES E OUTROS. MODO }\end{array}$ & 1902.20 .00 \\
\hline
\end{tabular}




\begin{tabular}{|c|c|c|}
\hline CAPÍTULO & PRODUTOS & CóDIGOS \\
\hline $\begin{array}{l}20 \text { - PREPARAÇÕES DE PRODUTOS } \\
\text { HORTÍCOLAS, DE FRUTAS ETC. }\end{array}$ & & 20 \\
\hline \multirow{4}{*}{$\begin{array}{c}21 \text { - PREPARAÇÕES ALIMENTÍCIAS } \\
\text { DIVERSAS }\end{array}$} & $\begin{array}{c}\text { KETCHUP E OUTROS MOLHOS DE TOMATE, EM- } \\
\text { BALAGENS IMEDIATAS, PESO }<=1 \mathrm{KG}\end{array}$ & 2103.20.10 \\
\hline & $\begin{array}{c}\text { MOSTARDA PREPARADA, EM EMBALAGENS IME- } \\
\text { DIATAS, PESO <= } 1 \mathrm{KG} \\
\end{array}$ & 2103.30.21 \\
\hline & OUTRAS MOSTARDAS PREPARADAS & 2103.30 .29 \\
\hline & $\begin{array}{l}\text { MAIONESE EM EMBALAGENS IMEDIATAS, PESO } \\
\qquad=1 \mathrm{KG}\end{array}$ & 2103.90.11 \\
\hline $\begin{array}{l}22 \text { - BEBIDAS, LÍQUIDOS ALCOÓLI- } \\
\text { COS E VINAGRES }\end{array}$ & CERVEJAS DE MALTE & 2203.00 .00 \\
\hline $\begin{array}{l}33 \text { - ÓLEOS ESSENCIAIS E RESINOI- } \\
\text { DES, PRODUTOS DE PERFUMARIA } \\
\text { ETC. }\end{array}$ & & 33 \\
\hline $\begin{array}{l}71 \text { - PÉROLAS NATURAIS OU CULTI- } \\
\text { VADAS, PEDRAS PRECIOSAS ETC. }\end{array}$ & & 71 \\
\hline \multirow{9}{*}{$\begin{array}{l}87 \text { - VEÍCULOS AUTOMÓVEIS, TRA- } \\
\text { TORES ETC. SUAS PARTES/ACESSÓ- } \\
\text { RIOS }\end{array}$} & $\begin{array}{l}\text { VEÍCULOS AUTOMÓVEIS PARA DESLOCAMENTO } \\
\text { NA NEVE, CAMPO GOLFE ETC. }\end{array}$ & 8703.10 .00 \\
\hline & $\begin{array}{l}\text { AUTOMÓVEIS COM MOTOR EXPLOSÃO, CIL <= } \\
1000 \mathrm{CM}^{3}\end{array}$ & 8703.21 .00 \\
\hline & AUTOMÓVEIS COM MOTOR EXPLOSÃO, 1000 & 8703.22 .10 \\
\hline & AUTOMÓVEIS COM MOTOR EXPLOSÃO, 1500 & 8703.23 .10 \\
\hline & AUTOMÓVEIS COM MOTOR DIESEL, 1500 & 8703.32 .10 \\
\hline & $\begin{array}{l}\text { AUTOMÓVEIS COM MOTOR DIESEL, CM3 > 2500, } \\
\text { ATÉ } 6 \text { PASSAGEIROS }\end{array}$ & 8703.33 .10 \\
\hline & $\begin{array}{c}\text { MOTOCICLETAS ETC. COM MOTOR PISTÃO ALTER- } \\
\text { NAT. } 250<\mathrm{C}<=500 \mathrm{CM}^{3}\end{array}$ & 8711.30 .00 \\
\hline & $\begin{array}{c}\text { MOTOCICLETAS ETC. COM MOTOR PISTÃO ALTER- } \\
\text { NAT. } 500<\mathrm{C}<=800 \mathrm{CM}^{3}\end{array}$ & 8711.40 .00 \\
\hline & $\begin{array}{l}\text { MOTOCICLETAS ETC. COM MOTOR PISTÃO ALTER- } \\
\text { NAT. CIL> } 800 \mathrm{CM}^{3} \\
\end{array}$ & 8711.50 .00 \\
\hline \multirow{7}{*}{$\begin{array}{l}88 \text { - AERONAVES E OUTROS APARE- } \\
\text { LHOS AÉREOS ETC. E SUAS PARTES }\end{array}$} & HELICÓPTEROS DE PESO <= 2000 KG, VAZIOS & 8802.11 .00 \\
\hline & HELICÓPTEROS DE 2000 KG & 8802.12 .10 \\
\hline & AVIÕES A HÉLICE ETC. PESO <= 2000 KG, VAZIOS & 8802.20 .10 \\
\hline & $\begin{array}{l}\text { AVIÕES A TURBOÉLICE ETC. MONOMOTORES, P } \\
<=2000 \mathrm{KG} \text {, VAZIOS }\end{array}$ & 8802.20 .21 \\
\hline & 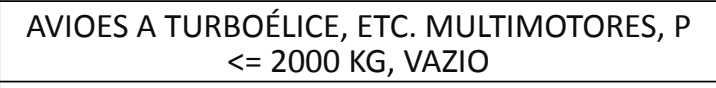 & 8802.20 .22 \\
\hline & AVIÕES A HÉLICE ETC. 2000 KG & 8802.30 .10 \\
\hline & AVIÕES A TURBOÉLICE ETC. MULTIMOTORES, 2T & 8802.30 .21 \\
\hline \multirow{3}{*}{$\begin{array}{c}89 \text { - EMBARCAÇÕES E ESTRUTURAS } \\
\text { FLUTUANTES }\end{array}$} & BARCOS A VELA, MESMO COM MOTOR AUXILIAR & 8903.91 .00 \\
\hline & $\begin{array}{l}\text { BARCOS A MOTOR, EXC. COM MOTOR FORA-DE } \\
\text {-BORDA }\end{array}$ & 8903.92 .00 \\
\hline & $\begin{array}{c}\text { OUTROS BARCOS/EMBARCAÇÕES DE RECREIO/ } \\
\text { ESPORTE, INCLUINDO CANOAS }\end{array}$ & 8903.99 .00 \\
\hline $\begin{array}{l}91 \text { - RELÓGIOS E APARELHOS SE- } \\
\text { MELHANTES, E SUAS PARTES }\end{array}$ & $\begin{array}{l}\text { RELÓGIO DE PULSO, DE BOLSO E SEMELHANTES } \\
\text { C/ CX. DE METAIS PRECIOSOS OU METAIS FOLHE- } \\
\text { ADOS OU CHAPEADOS DE METAIS PRECIOSOS. }\end{array}$ & 91 \\
\hline
\end{tabular}




\begin{tabular}{|c|c|c|}
\hline CAPÍTULO & PRODUTOS & CódIGOS \\
\hline $\begin{array}{c}94-\text { MÓVEIS, MOBILIARIO MÉDI- } \\
\text { CO-CIRÚRGICO, COLCHÕES ETC. }\end{array}$ & MÓVEIS DE METAL PARA ESCRITÓRIOS & 94 \\
\hline $\begin{array}{c}95-\text { BRINQUEDOS, JOGOS, ARTI- } \\
\text { GOS P/DIVERTIMENTO, ESPORTES } \\
\text { ETC. }\end{array}$ & $\begin{array}{c}\text { BRINQUEDOS, JOGOS, ARTIGOS PARA DIVERTI- } \\
\text { MENTO, ESPORTES ETC. }\end{array}$ & 95 \\
\hline $\begin{array}{c}97 \text { - OBJETOS DE ARTE, DE COLE- } \\
\text { ÇÃO E ANTIGUIDADES }\end{array}$ & $\begin{array}{c}\text { QUADROS, PINTURAS E DESENHOS, FEITOS A } \\
\text { MÃOO }\end{array}$ & 97 \\
\hline
\end{tabular}

Nota: Fonte: Adaptado a partir da tabela de produtos da NCM. Ministério do Desenvolvimento, Indústria e Comércio Exterior 